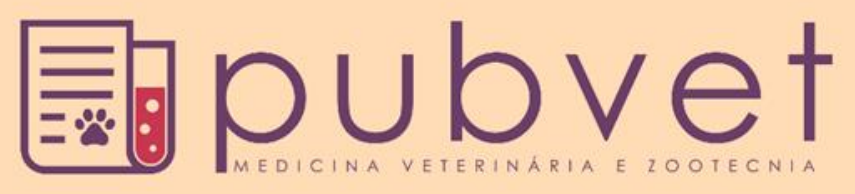

ISSN $1982-1263$

https://doi.org/10.31533/pubvet.v13n6a347.1-9

\title{
Rhipicephalus (Boophilus) microplus e Rhipicephalus sanguíneus: uma revisão sobre as perspectivas, distribuição e resistência
}

\author{
Tamara de Moura Mendes ${ }^{1 *} \bullet$, Jéssica Nery Ferreira Balbino ${ }^{1}$, Noemi Cunha Torres \\ Silva $^{1}$, Leonardo Alves de Farias ${ }^{20}$
}

${ }^{1}$ Acadêmica da Faculdade Maurício de Nassau, Departamento de Medicina Veterinária. João Pessoa - PB Brasil.

${ }^{2}$ Docente da Faculdade Maurício de Nassau. João Pessoa, PB e Brasil

*autor para correspondência, E-mail: tamis_pbjp@yahoo.com.br

\begin{abstract}
Resumo. Desde o início dos tempos a produção animal é diretamente influenciada por parasitas. Dentre estes está o ectoparasita dos bovinos, o carrapato Ripicephalus (Boophilus) microplus. O controle destes ácaros é feito através de manejo convencional com produtos sintéticos, o que se torna problemático no ponto de vista da resistência destes carrapatos, refletindo diretamente em um número excessivo de aplicações aliadas a dosagens acima do recomendado, em intervalos menores do que o necessário. Desta forma está crescendo o número de investigações científicas que objetivam o desenvolvimento de produtos menos agressivos e mais sustentáveis. Não menos preocupante, o Rhipicephalus sanguineus, além de ser um dos principais problemas parasitários enfrentados por proprietários de canis, também tem sido encontrado no ambiente domiciliar e peridomiciliar do homem que convive com o seu principal hospedeiro urbano, o cão doméstico. A espécie de carrapato $R$. sanguineus tem sido encontrada com alta prevalência em cães urbanos, sendo descrita na literatura como uma das principais espécies de parasitas destes animais domésticos. Espécie comprovadamente envolvida na transmissão de agentes patogênicos, destacando - se entre estes: Babesia canis, Eherlichia canis e Anaplasma platys. Este ácaro está se tornando uma verdadeira praga urbana digna de atenção dos órgãos de saúde pública, e motivo de grande preocupação entre os profissionais veterinários.
\end{abstract}

Palavras chave: boi, cão, carrapato, ectoparasita, vetor

\section{Rhipicephalus (Boophilus) microplus and Rhipicephalus sanguineus: a review on the prospects, distribution and resistance}

Abstract. Since the beginning of time animal production is directly influenced by parasites.
Among these are the ectoparasites bovine, the tick Ripicephalus (Boophilus) microplus.
The control of these mites is done through conventional management with synthetic
products, which becomes problematic in the point of view of the resistance of these ticks,
directly reflecting in an excessive number of applications allied to dosages above the
recommended ones, in smaller intervals than necessary. In this way the number of scientific
investigations aiming at the development of less aggressive and more sustainable products
is growing. Not less worrisome, Rhipicephalus sanguineus, besides being one of the main
parasitic problems faced by kennel owners, has also been found in the home and peri-
domiciliary environment of the man who lives with his main urban host, the domestic dog.
The R. sanguineus tick species has been found with high prevalence in urban dogs, being
described in the literature as one of the main parasite species of these domestic animals.
Species evidently involved in the transmission of pathogenic agents, such as Babesia canis,
Eherlichia canis and Anaplasma platys. This mite is becoming a real urban pest worthy of 
attention of the public health organs, and cause for great concern among veterinary professionals.

Keywords: dog, ectoparasite, teer, ticks, vector

\title{
Rhipicephalus (Boophilus) microplus y Rhipicephalus sanguíneus: una revisión sobre las perspectivas, la distribución y la resistencia
}

\begin{abstract}
Resumen. Desde el inicio de los tiempos la producción animal es directamente influenciada por parásitos. Entre estos está el ectoparásito de los bovinos, la garrapata Ripicephalus (Boophilus) microplus. El control de estos ácaros se realiza a través de manejo convencional con productos sintéticos, lo que se vuelve problemático desde el punto de vista de la resistencia de estas garrapatas, reflejando directamente en un número excesivo de aplicaciones aliadas a dosificaciones arriba de lo recomendado, a intervalos menores de lo necesario. De esta forma está creciendo el número de investigaciones científicas que objetivan el desarrollo de productos menos agresivos y más sostenibles. No es menos preocupante, el Rhipicephalus sanguineos, además de ser uno de los principales problemas parasitarios enfrentados por propietarios de canis, también ha sido encontrado en el ambiente domiciliar y peridomiciliar del hombre que convive con su principal huésped urbano, el perro doméstico. La especie de garrapata $R$. sanguineus ha sido encontrada con alta prevalencia en perros urbanos, siendo descrita en la literatura como una de las principales especies de parásitos de estos animales domésticos. Especie comprobadamente involucrada en la transmisión de agentes patógenos, destacándose entre éstos: Babesia canis, Eherlichia canis y Anaplasma platys. Este ácaro se está convirtiendo en una verdadera plaga urbana digna de atención de los órganos de salud pública, y motivo de gran preocupación entre los profesionales veterinarios.
\end{abstract}

Palabras clave: buey, ectoparásito, garrapata, perro, vector

\section{Introdução}

O Rhipicephalus (Boophilus) microplus e o Rhipicephalus sanguineus são carrapatos da ordem dos ixodideos associados à disseminação de diversas e importantes doenças nos bovinos e caninos respectivamente. O primeiro sendo monóxeno, ou seja, carrapato de um só hospedeiro, embora presente o ano todo, tem seu maior pico no verão. Todos os estágios do parasita podem transmitir doenças, inclusive por via transovariana, diferentemente de $R$. sanguineus. Este por sua vez é um carrapato trioxeno, ou seja, necessita de três hospedeiros para completar seu ciclo. Muitas gerações podem ocorrer durante o ano, principalmente durante o verão (Taylor et al., 2017).

O ectoparasitismo é considerado um dos maiores problemas da atividade pecuária no Brasil, com destaque para o carrapato bovino R. (Boophilus) microplus, sendo este o mais importante das Américas do Sul, Central, África e Oceania (Andreotti, 2010; Horn \& Arteche, 1985; Silva \& Rocha, 2004).

Com o objetivo de controlar este parasita e os prejuízos por ele causados, o produtor rural utiliza produtos químicos convencionais à base de piretróides, formamidinas, lactonasmacrocíclicas, tiazolidinas, organofosforados e fenilureias. Porém, na maioria das vezes essa estratégia não é efetiva e, principalmente sustentável, já que as dosagens empregadas são maiores que a recomendada, criando problemas relacionados à resistência, e embora estejam sendo desenvolvidos vários novos produtos, estes ainda não são isentos do risco de resistência, sem mencionarmos o fato de a aplicação não seguir as normas básicas de segurança na aplicação de agrotóxicos (Agnolin et al., 2010; Bullman et al., 1996; Klafke et al., 2006).

Incluem-se ainda os problemas de saúde humana, já que a maioria dos produtores rurais não utilizam equipamentos de segurança do trabalho adequados nem respeitam os períodos de carência dos produtos utilizados no controle de ectoparasitos, principalmente no que se diz respeito à contaminação da carne e do leite por resíduos químicos (Agnolin et al., 2010; Bullman et al., 1996; Klafke et al., 2006). Quanto à espécie Rhipicephalus sanguineus, é certamente o ectoparasita de mais ampla distribuição no mundo, podendo ser encontrado em todos os continentes habitados por humanos e cães domésticos (Walker et 
al., 2005). Mesmo havendo relatos de parasitismo por $R$. sanguineus em diversas espécies animais e em diferentes regiões geográficas, o cão doméstico é notoriamente o principal hospedeiro para todos os estágios parasitários deste carrapato (Walker et al., 2005). Rhipicephalus sanguineus é considerado um dos carrapatos de maior importância médico-veterinário mundial, pois além dos danos diretos causados pelo parasitismo propriamente dito, que não muito raramente causa anemia grave em cães, é também vetor natural da bactéria Ehrlichia canis, dos protozoários Babesia canis, B. gibsoni, Anaplasma platys e Hepatozoon canis (Baneth et al., 2001; Gothe et al., 1989; Groves et al., 1975; Smith et al., 1976).

Existe a discussão de que $R$. sanguineus também é vetor natural de Leishmania (Leishmania) infantum, e está envolvido na transmissão de riquétsias para humanos na Europa (especialmente Rickettsiaconorii) (Bustamante \& Varela, 1947; Demma et al., 2006).

\section{R. (Boophilus) microplus}

\section{Características morfológicas}

Esse carrapato possui a base do gnatossoma hexagonal com rostro, ou seja, hipostômio e quelíceras com palpos curtos, com o terceiro segmento do palpo fazendo uma aba na junção com o segundo segmento, possui seu Escudo sem ornamentação, os olhos estão presentes e os festões ausentes, além de estigmas arredondados ou ovais. Os machos apresentam dois pares de placas adanais desenvolvidas, geralmente com prolongamento caudal e são menores que as fêmeas (Monteiro, 2011).

\section{Distribuição}

Esse ectoparasita é originário do sul da Ásia, onde foi encontrado parasitando principalmente artiodátilos nativos (Hoogstraal, 1979). Com o comércio de gado zebuíno do continente asiático para outros continentes, este ixodídeo, atualmente, está presente no hemisfério sul (Lombardo, 1975). Além de outros países, no território brasileiro encontram-se, em sua maioria, como área potencial ao desenvolvimento de $R$. (B.) microplus, possuindo presença importante na rotina da pecuária nacional (Horn \& Arteche, 1985).

A espécie de carrapatos mais presente em bovinos no Brasil é Boophilus microplus, mais conhecido como "O carrapato do boi", que a partir de 2003 passou a ser chamado Rhipicephalus (Boophilus) microplus, depois do uso da biologia molecular, porém ainda pode-se utilizar o nome Boophilus microplus (Godoi \& Silva, 2009). O carrapato dos bovinos encontra-se amplamente distribuído no Brasil, o qual possui o maior rebanho bovino comercial mundial, estimado em 200 milhões de animais, ele é a principal forma de transmissão de patologias de alta morbidade e mortalidade como a anaplasmose e a babesiose bovina. Essas doenças também estão associadas aos prejuízos marcados pela infestação por carrapatos, os quais causam diminuição na produção, anemia e até mesmo causar o óbito de bovinos (Cordovés, 1997). A presença do $R$. (B.) microplus é confirmada em todo o Brasil, sendo principalmente encontrado durante o ano todo em 66,0\% dos municípios (Horn \& Arteche, 1985; Silva \& Rocha, 2004). Estudos que buscam a avaliação do abalo econômico que a ação dos ectoparasitos na América do Sul pode causar consideraram que no Brasil, as perdas chegam a 2,5 milhões de cabeças de gado, o que representa a perca de 75 milhões de quilogramas de carne, 1,5 bilhão de litros de leite, 8,6 milhões de dólares por danos secundários e 25 milhões de dólares em acaricidas químicos para combater as infestações por carrapatos (Gomes, 1998).

Na Austrália, as perdas por ano na cadeia de produção devido aos carrapatos são de, em média, US\$ 42 milhões. Para o México, as estimativas são de US\$ 3,05 bilhões anual. No Brasil, os prejuízos atribuídos ao carrapato ficam próximos a oito dólares/bovino/ano, podendo, ultrapassar um bilhão de dólares anuais (Gomes, 1998).

\section{Resistência}

A resistência aos pesticidas significa uma resposta evolutiva do agente contra sua eliminação. $\mathrm{O}$ tratamento com pesticidas elimina aqueles indivíduos da população que são suscetíveis, contribuindo com a manutenção daqueles que se tornaram resistentes. Quanto maior o número de aplicações dessas drogas, mais indivíduos resistentes são selecionados, diminuindo cada vez mais a eficácia dos pesticidas, ocasionando assim a falha no controle. A administração incorreta dos carrapaticidas, a exemplo de 
superdose e/ou excesso de aplicações, provocam a resistência do carrapato às drogas as quais o mercado tem disponível (Le Gall et al., 2018). Os primeiros registros de resistência do Rhipicephalus microplus ao arsênio no Brasil datam de 1953 (Freire, 1953). A resistência foi confirmada para organoclorados em 1953 (Martin et al., 2003), organofosforados em 1963 (Furlong, 1999), piretroides sintéticos em 1989 (Arantes et al., 1995), amidinas em 1995 (Martins et al., 1995) e lactonasmacrocíclicas em 2001 (Klafke et al., 2006; Martin et al., 2003; Martins \& Furlong, 2001).

É necessário o conhecimento dos produtos os quais os carrapatos já desenvolveram resistência, para que assim, seu uso possa ser evitado. Atualmente existem seis variedades de carrapaticidas disponíveis no mercado para o controle do carrapato Rhipicephalus microplus (piretróides, organofosforados, amitraz, fipronil, fluazuron e lactonasmacrocíclicas). Todavia, alguns princípios ativos já não apresentam eficácia satisfatória, o que justifica o surgimento de populações resistentes. A droga de referência no combate ao carrapato $R$. microplus é a ivermectina, com propriedades anti-helmínticas, acaricidas e inseticidas. A ivermectina é um antiparasita de amplo espectro, indicado para combater os principais nematódeos e cestódeos (Le Gall et al., 2018).

Os meios fisiológicos utilizados pelo $R$. microplus que influenciam na resistência aos carrapaticidas se diferem de acordo com sua resposta ao princípio ativo usado nas drogas. Eles podem variar em alteração no comportamento do parasito para evitar contato com o produto; modificação no exoesqueleto para reduzir a penetração cuticular do princípio ativo; aumento da detoxificação metabólica e modificação do sítio de ação para redução na sensibilidade ao carrapaticida (Alonso-Díaz et al., 2006).

Drogas que têm como princípio ativo a ivermectina causa paralisia da musculatura e morte dos parasitas suscetíveis, ocasionado pelo aumento da liberação do neurotransmissor GABA (ácido gamaaminobutírico). A paralisia é advogada pelo potencial de ação dos canais de cloro sensíveis à ivermectina, controlados pelo glutamato. Esses canais estão presentes somente nos nervos e células musculares dos invertebrados, que quando ativos, ocasionam um aumento da permeabilidade da membrana celular aos íons cloreto, com hiperpolarização dos nervos ou células musculares, resultando na paralisia e em seguida na morte do parasita (Le Gall et al., 2018). De acordo com a pesquisa, em $R$. microplus diversos mecanismos podem estar envolvidos na resistência à ivermectina, incluindo a capacidade fisiológica de detoxificação. Os mecanismos metabólicos da desintoxicação são mediados por famílias de enzimas, incluindo o citocromo P450, esterase e glutationa-S-transferases (GST) e também por proteínas específicas, como os transportadores ABC, contribuem para os processos de desintoxicação, transportando substâncias tóxicas para fora das células, com consumo de ATP. Essa competência fisiológica de produção de defesa do organismo é tida como seleção natural (Le Gall et al., 2018).

Ademais, o uso de substâncias homeopáticas tem sido uma possibilidade para controle dos carrapatos, haja que não existem evidências de que esses produtos possam provocar resistência nos mesmos. Os estudos com as plantas ainda estão no início. Todavia, trabalhos que utilizaram óleos essenciais e concentrados emulsionáveis de eucalipto (Eucalyptus) e rotenóides extraídos do timbó, (Derris urucu) mostraram-se promissores no controle desse ácaro. Em contrapartida, o Nim (Azadirachta indica), planta com ação inseticida, testada na forma de óleo, extrato alcoólico ou aquoso, teve baixa ou nenhuma eficácia sobre fêmeas ingurgitadas e larvas do carrapato em ensaios de laboratório (Silveira et al., 2014).

\section{Perspectivas}

No Brasil, várias equipes têm trabalhado com o emprego de fungos para controlar $R$ (Boophilus) microplus. Os resultados obtidos nos ensaios de laboratório foram muito bons, porém os resultados alcançados a campo (Bahiense et al., 2007; Bittencourt et al., 1999; Castro et al., 1997; Correia et al., 1998), ou no pasto, não foram muito promissores. O fungo da espécie Metarhiziumanisopliae tem sido um dos mais estudados com potencial de controle biológico do carrapato em todo o mundo (Bittencourt et al., 2003; Garcia et al., 2011). No que foi observado, o uso destes fungos colocou como desvantagens a demora em exterminar os carrapatos, além da necessidade de umidade para germinação e esporulação, a vulnerabilidade à radiação ultravioleta, e da possibilidade de algumas cepas afetarem o desenvolvimento de outros organismos. Ojeda-Chi et al. (2011) realizaram uma série de revisões bibliográfica sobre a relação deste fungo com o ixodideo Rhipicephalus microplus, ressaltando aspectos importantes, como particularidades relativas à penetração do fungo no carrapato, o motivo da morte do 
carrapato, sua consequência em outros artrópodes relevantes na veterinária e também analisam os resultados de pesquisas efetuadas in vitro e in vivo. Após estudos em laboratório concluíram que quando o fungo $M$. anisopliae foi aplicado juntamente com carrapaticida de base piretroide em larvas de $R$. microplus resistentes a esse produto, ocorreu aumento da mortalidade das larvas, chegando a índices de quase $100 \%$, indicando que a junção do fungo com o carrapaticida pode vir a ser uma estratégia de relevante para o controle integrado do carrapato $R$ microplus (Bahiense et al., 2006).

Outras espécies de fungos foram encontradas infectando carrapatos e estudadas quanto ao uso potencial no seu controle. Alecanicillium lecanii foi estudada por Angelo et al. (2010) que analisaram seu impacto sobre larvas e fêmeas ingurgitadas deste carrapato, in vitro. Os resultados indicaram que o fungo é favorável no controle biológico desta espécie.

Entre os animais vertebrados, o maior inimigo do carrapato é o próprio bovino, já que apresenta níveis distintos de resistência ao parasita. É notório que os bovinos de raças zebuínas são mais resistentes à $R$ microplus, com menor número e tamanho de teleóginas que os das raças taurinas (Villares, 1941). Essas larvas têm certa dificuldade em se fixar e completar o ciclo em bovinos resistentes, o que as torna menores e mais leves, causando imenso impacto epidemiológico. Rocha (2014) mencionou que fêmeas retiradas de bovinos resistentes são mais leves, portanto, diminuindo também a postura de ovos, consequentemente originando menos larvas do que aquelas retiradas de hospedeiros predispostos ao parasita. O número de ovos postos por uma teleógina é proporcional ao peso que podem atingir, o que afeta diretamente a capacidade de consumo de sangue do hospedeiro (Rocha, 2014; Rocha et al., 1984; Santos \& Furlong, 2002) e uma parte dos ovos sofrem dessecação, diminuindo a quantidade de larvas viáveis (Moraes et al., 1989).

Em gado de corte, foi observado que novilhas mestiças recém-desmamadas (8-9 meses de idade) $1 / 2$ Angus $\mathrm{x} 1 / 2$ Nelore criadas nas pastagens, engordaram mesmo possuindo mais carrapatos que novilhas Nelore e Guzerá, sem, contudo necessitarem de carrapaticida por pelo menos 11 meses, demonstrando que o cruzamento de uma raça europeia própria para a produção de carne, com a raça Nelore, pode ser uma estratégia sustentável e econômica para o aumento da produção de carne no Brasil (Marini et al., 2010).

\section{Rhipicephalus Sanguineus}

\section{Características morfológicas}

Esta espécie de carrapato tem coloração amarela, vermelhada ou castanho e apresenta como principal característica o estigma na forma de vírgula, observado principalmente no macho e menos acentuado na fêmea, olhos e festões estão presentes. Seu tamanho pode variar nas fêmeas ingurgitadas que chegam ao comprimento de $12 \mathrm{~mm}$. Os palpos são curtos e a base do capítulo é dorsalmente hexagonal, a coxa do primeiro par de pernas apresenta dois espinhos e as pernas podem se tornar ligeiramente maiores do par anterior para o par posterior. O sulco anal circunda a metade posterior do ânus e se estende para o sulco mediano. Os carrapatos machos apresentam placas adanais e escudos acessórios. As larvas de seis pernas são pequenas e de coloração castanho-claro enquanto as ninfas de oito pernas têm coloração castanha avermelhada (Taylor et al., 2017).

\section{Distribuição}

A espécie Rhipicephalus sanguineus (Latreille) é provavelmente o carrapato de maior distribuição mundial, estando presente em todos os continentes habitados por humanos e cães domésticos (Walker et al., 2005). Com origem no continente africano, $R$. sanguineus é atualmente encontrado em todos os países da América do Norte e do Sul, onde foi incorporado e estabelecido, juntamente com cães domésticos, em períodos antes e/ou após a colonização europeia (Labruna, 2004). Embora haja muitos relatos de parasitismo por $R$. sanguineus em diversas e diferentes espécies de animais em distintas regiões geográficas, o cão doméstico é o principal hospedeiro para todos os estágios parasitários deste ectoparasita (Walker et al., 2005). Apesar de não ser muito comum o parasitismo humano por $R$. sanguineus, casos tem sido relatados mundialmente, inclusive no Brasil e América do Norte (DantasTorres et al., 2006; Goddard, 1989). 
No início do século $\mathrm{XX}, R$. sanguineus era assinalado como um carrapato de distribuição restrita a apenas alguns estados brasileiros (Aragão, 1911; Aragão \& Fonseca, 1936). Com o passar dos anos, ele foi tornando-se de distribuição mais ampla, e mais recentemente, mais abundante (Labruna, 2004). Atualmente, a espécie está presente em todo o território brasileiro, principalmente nas áreas urbanas (Labruna, 2004; Labruna et al., 2000).

\section{Resistência e perspectivas}

O carrapato Rhipicephalus sanguineus, espécie que acomete cães - tem o mesmo como seu hospedeiro definitivo. Esse ectoparasita é eventualmente um agente patogênico, o que tem despertado o interesse na saúde pública pela transmissão de doenças aos humanos, contingentemente, seu hospedeiro acidental. Em área urbana, o $R$. sanguineus além de ser um infortúnio para donos de canis, é também um contratempo encontrado em cães domésticos (Paz et al., 2008). Para um efetivo controle do Rhipicephalus Sanguineus é fundamental o uso de pesticidas os quais o carrapato seja suscetível. No Brasil, não há estudos sobre a resistência deste carrapato com base em comparações com uma cepa sensível, e mesmo mundialmente há poucos relatos (Borges et al., 2007).

Atualmente são encontrados no mercado medicamentos antiparasitários administrados tanto por via oral em cães, como também por aplicação cutânea, a exemplo de fipronil e fluralaner, dentre outros existentes. Em um estudo de caso, foi comparada à eficácia do controle de pulgas e carrapatos em cães durante um período de 12 semanas com uma única dose administrada por via oral de fluralaner (Bravecto ${ }^{\text {MR }}$ ), formulado como um comprimido mastigável ou com três tratamentos tópicos sequenciais de fipronil (Frontline ${ }^{\mathbf{M R}}$ ) (Rohdich et al., 2014). Foi testada em uma pesquisa de campo, com duração de dose semanas, a eficácia de duas substâncias relacionadas ao controle de carrapatos e pulgas. Nas semanas 2, 4, 8 e 12, a eficácia de controle de pulgas em domicílios tratados com fluralaner (Bravecto ${ }^{\mathbf{M R}}$ ) foi de $99,2 \%, 99,8 \%, 99,8 \%$ e 99,9\%, respectivamente, enquanto com fipronil (Frontline ${ }^{\mathbf{M R}}$ ), a eficiência do produto foi de $94,1 \%, 93,0 \%, 96,0 \%$, e $97,3 \%$, na devida ordem. A eficácia do controle de carrapatos com fluralaner sobre cães tratados nas semanas 2, 4, 8 e 12 foi de 99,9\%, 99,9\%, 99,7\% e 100\%, sendo o do fipronil de $97,6 \%, 93,8 \%, 100 \%$ e 100\%, respectivamente (Rohdich et al., 2014). Pôde-se concluir que, para o controle do carrapato Rhipicephalus sanguineus, o fluralaner propinado em dose única por via oral nos cães foi sobremodo eficaz durante dose semanas contra pulgas e carrapatos, e foi significativamente não inferior (carrapatos) e superior (pulgas) em referência à administração tópica de fipronil, aplicada por três vezes seguidas (Rohdich et al., 2014).

\section{Conclusão}

A criação de bovinos resistentes ao carrapato é a forma mais eficaz (controle efetivo da população de carrapatos), econômica (não há gastos com insumos para seu controle) e ecológica (sem resíduos para o ambiente) atualmente usada no controle do carrapato $R$. microplus. Na pecuária de corte o problema do carrapato é minimizado com a criação de raças zebuínas, e os cruzamentos de animais resistentes (zebuínos) com suscetíveis (europeus), assim como a criação da raça Jersey, contribuem para o controle biológico do carrapato na pecuária de corte e leiteira.

É notório também que um conjunto de fatores define o sucesso biológico de $R$. sanguineus e dentre eles podemos citar sua ampla distribuição geográfica, poucos inimigos naturais, elevados níveis de infestação, assim como mudanças climáticas, desmatamentos, que sem dúvida vem favorecendo o maior contato com os hospedeiros alvos (cão).

Assim sendo, a ampla adaptação desses artrópodes aos diferentes ambientes, tem contribuído para sua proliferação exponencial, o que provoca a necessidade de se buscar estratégias de controle que sejam eficientes e ao mesmo tempo sustentáveis na tentativa de minimizar os danos colaterais causados pelos acaricidas químicos.

\section{Referências bibliográficas}

Agnolin, C. A., Olivo, C. J., Leal, M. L. R., Beck, R. C. R., Meinerz, G. R., Parra, C. L. C. \& Nicolodi, P. R. S. J. (2010). Efficacy of citronella [Cymbopogon nardus (L.) Rendle] oil in the control of bovine ectoparasites. Revista Brasileira de Plantas Medicinais, 12(4):482-487. 
Alonso-Díaz, M. A., Rodríguez-Vivas, R. I., Fragoso-Sánchez, H. \& Rosario-Cruz, R. (2006). Resistencia de la garrapata Boophilus microplus a los ixodicidas. Archivos de Medicina Veterinaria, 38(2):105-113.

Andreotti, R. (2010). Situação atual da resistência do carrapato-do-boi Rhipicephalus (Boophilus) microplus aos acaricidas no Brasil. Campo Grande, Mato Grosso do Sul, Brasil: Embrapa Gado de Corte-Documentos.

Angelo, I. C., Fernandes, É. K. K., Bahiense, T. C., Perinotto, W. M. S., Moraes, A. P. R., Terra, A. L. M. \& Bittencourt, V. R. E. P. (2010). Efficiency of Lecanicillium lecanii to control the tick Rhipicephalus microplus. Veterinary Parasitology, 172(3-4):317-322.

Aragão, H. B. (1911). Notas sobre ixódidas brazileiros. Memórias do Instituto Oswaldo Cruz, 3(2):145195.

Aragão, H. B. \& Fonseca, F. (1936). Ixodidas brasileiros e de alguns paizes limitrophes. Memórias do Instituto Oswaldo Cruz, 31(4):759-843.

Arantes, G. J., Marques, A. O. \& Honer, M. R. (1995). O carrapato do bovino, Boophilus microplus, no município de Uberlândia, MG: Análise da sua resistência contra carrapaticidas comerciais. Revista Brasileira de Parasitologia Veterinária, 4(2):89-93.

Bahiense, T. C., Fernandes, É. K. K., Angelo, I. C., Perinotto, W. M. S. \& Bittencourt, V. R. (2007). Avaliação do potencial de controle biológico do Metarhizium anisopliae sobre Boophilus microplus em teste de estábulo. Revista Brasileira de Parasitologia Veterinária, 16(4):243-245.

Bahiense, T. C., Fernandes, É. K. K. \& Bittencourt, V. R. E. P. (2006). Compatibility of the fungus Metarhizium anisopliae and deltamethrin to control a resistant strain of Boophilus microplus tick. Veterinary Parasitology, 141(3-4):319-324.

Baneth, G. A. D., Samish, M., Alekseev, E., Aroch, I. \& Shkap, V. (2001). Transmission of Hepatozoon canis to dogs by naturally-fed or percutaneously-injected Rhipicephalus sanguineus ticks. Journal of Parasitology, 87(3):606-612.

Bittencourt, V. R. E. P., Bahiense, T. C., Fernandes, E. K. K. \& Souza, E. J. (2003). Avaliação da ação in vivo de Metarhizium anisopliae (Metschnikoff, 1879) Sorokin, 1883 aplicado sobre Brachiaria decumbens infestada com larvas de Boophilus microplus (Canestrini, 1887) (Acari: Ixodidae). Revista Brasileira de Parasitologia Veterinária, 12(1):38-42.

Bittencourt, V. R. E. P., Souza, E. J., Peralva, S. L. F. S. \& Reis, R. C. S. (1999). Eficácia do fungo Metarhizium anisopliae (Metschnikoff, 1879) Sorokin, 1883 em teste de campo com bovinos infestados por carrapato Boophilus microplus (Canestrini, 1887) (Acari: Ixodidae). Revista Brasileira de Medicina Veterinária, 2178-82.

Borges, L. M. F., Soares, S. F., Fonseca, I. N., Chaves, V. V. \& Louly, C. C. B. (2007). Resistência acaricida em larvas de Rhipicephalus sanguineus (Acari: Ixodidae) de Goiânia-GO, Brasil. Revista de Patologia Tropical, 36(1):87-95.

Bullman, G. M., Muños Cabenas, M. E. \& Ambrústolo, R. R. (1996). El impacto ecológico de las lactonas macrociclicas (endectocidas): una actualizacion compreensiva y comparativa. Veterinária Argentina, 8(127):3-15.

Bustamante, M. E. \& Varela, G. (1947). Rhipicephalus sanguineus e a epidemiologia da leishmaniose visceral canina no Estado de Pernambuco. Revista del Instituto de Salubridade y Enfermedades Tropicales, 8(2):139-141.

Castro, A. B. A., Bittencourt, V. R. E. P., Daemon, E. \& Viegas, E. C. (1997). Eficácia do fungo Metarhizium anisopliae sobre o carrapato Boophilus microplus em teste de estábulo. Revista da Universidade Rural, Série Ciências da Vida, 1973-82.

Cordovés, C. O. (1997). Carrapato: controle ou erradicação (Vol. 1). Porto Alegre, Rio Grande do Sul: Guaíba Agropecuária.

Correia, A. d. C. B., Fiorin, A. C., Monteiro, A. C. \& Veríssimo, C. 1. J. (1998). Effects of metarhizium anisopliaeon the tick Boophilus microplus (Acari: Ixodidae) in Stabled Cattle. Journal of Invertebrate Pathology, 71(2):189-191. 
Dantas-Torres, F., Figueredo, L. A. \& Brandão-Filho, S. P. (2006). Rhipicephalus sanguineus (Acari: Ixodidae), the brown dog tick, parasitizing humans in Brazil. Revista da Sociedade Brasileira de Medicina Tropical, 39(1):64-67.

Demma, L. J., Eremeeva, M., Nicholson, W. L., Traeger, M., Blau, D., Paddock, C. \& Swerdlow, D. (2006). An outbreak of Rocky Mountain Spotted Fever associated with a novel tick vector, Rhipicephalus sanguineus, in Arizona, 2004: preliminary report. Annals of the New York Academy of Sciences, 1078(1):342-343.

Freire, J. J. (1953). Arseno e cloro resistência e emprego de tiofosfato de dietilparanitrofenila (Parathion) na luta anticarrapato Boophilus microplus (Canestrini, 1887). Boletim da Diretoria de Produção Animal, 9(17):3-21.

Furlong, J. (1999). Diagnosis of the susceptibility of the cattle tick, Boophilus microplus, to acaricides in Minas Gerais state, Brazil. Paper presented at the 4. Seminario Internacional de Parasitología Animal, Puerto Vallarta, México.

Garcia, M. V., Monteiro, A. C., Szabó, M. P. J., Mochi, D. A., Simi, L. D., Carvalho, W. M. \& Barbosa, J. C. (2011). Effect of Metarhizium anisopliae fungus on off-host Rhipicephalus (Boophilus) microplus from tick-infested pasture under cattle grazing in Brazil. Veterinary Parasitology, 181(24):267-273.

Goddard, J. (1989). Focus of human parasitism by the brown dog tick, Rhipicephalus sanguineus (Acari: Ixodidae). Journal of Medical Entomology, 26(6):628-631.

Godoi, C. R. \& Silva, E. F. P. (2009). Carrapato Boophilus microplus e impacto na produção animalRevisão de literatura. PUBVET, 3(22):Art 606.

Gomes, A. (1998). Controle do carrapato do boi: um problema para quem cria raças européias. Embrapa Gado de Corte, 31(1):1-8.

Gothe, R., Wegerdt, S., Walden, R. \& Walden, A. (1989). On the epidemiology of infections with Babesia canis and Babesia gibsoni in dogs in Germany. Kleintierpraxis, 34(7):309-320.

Groves, M. G., Dennis, G. L., Amyx, H. L. \& Huxsoll, D. L. (1975). Transmission of Ehrlichia canis to dogs by ticks (Rhipicephalus sanguineus). American Journal of Veterinary Research, 36(7):937-940.

Hoogstraal, H. (1979). The epidemiology of tick-borne Crimean-Congo hemorrhagic fever in Asia, Europe, and Africa. Journal of Medical Entomology, 15(4):307-417.

Horn, S. C. \& Arteche, C. C. P. (1985). Situação parasitária da pecuária no Brasil. A Hora Veterinária, 4(23):12-32.

Klafke, G. M., Sabatini, G. A., Thais, A., Martins, J. R., Kemp, D. H., Miller, R. J. \& Schumaker, T. T. S. (2006). Larval immersion tests with ivermectin in populations of the cattle tick Rhipicephalus (Boophilus) microplus (Acari: Ixodidae) from State of Sao Paulo, Brazil. Veterinary Parasitology, 142(3-4):386-390.

Labruna, M. B. (2004). Biologia-ecologia de Rhipicephalus sanguineus (Acari: ixodidae). Revista Brasileira de Parasitologia e Veterinária, 13(S1):123-124.

Labruna, M. B., Homem, V. S. F., Heinemann, M. B. \& Neto, J. S. F. (2000). Ticks (Acari: Ixodidae) associated with rural dogs in Uruará, eastern Amazon, Brazil. Journal of Medical Entomology, 37(5):774-776.

Le Gall, V. L., Klafke, G. M. \& Torres, T. T. (2018). Detoxification mechanisms involved in ivermectin resistance in the cattle tick, Rhipicephalus (Boophilus) microplus. Scientific Reports, 8(1):1-10.

Lombardo, R. A. (1975). Socio-economic importance of the tick problem in the Americas. Paper presented at the 8. Reunion Interamericana a Nivel Ministerial sobre el Control de la Fiebre Aftosa y Otras Zoonosis, Guatemala City.

Marini, A., Soutello, R. V. G., Costa, R. L. D., Neves, J. H., Vacati, A. C., Barreto, T. N. \& Demarchi, J. J. A. A. (2010). Infestação por Rhipicephalus (Boophilus) microplus em novilhas de diferentes grupos genéticos. Boletim de Indústria Animal, 67(1):65-71.

Martin, T., Ochou, O. G., Vaissayre, M. \& Fournier, D. (2003). Oxidases responsible for resistance to pyrethroids sensitize Helicoverpa armigera (Hübner) to triazophos in West Africa. Insect Biochemistry and Molecular Biology, 33(9):883-887. 
Martins, J. R., Correa, B. L., Ceresér, V. H. \& Arteche, C. C. P. A. (1995). A situation report on resistance to acaricides by the cattle tick Boophilus microplus in the state of Rio Grande do Sul, Southern Brazil. Paper presented at the Publicacion Especial, Acapulco, México.

Martins, J. R. \& Furlong, J. (2001). Avermectin resistance of the cattle tick Boophilus microplus in Brazil. The Veterinary Record, 149(2):64-64.

Monteiro, S. G. (2011). Parasitologia na medicina veterinária (Vol. 1). São Paulo: Roca.

Moraes, F. R., Rocha, U. F., Costa, A. J., Moraes, J. R. E., Banzatto, D. A. \& Vasconcelos, O. T. (1989). Ecologia de carrapatos XXV-Correlação entre a resistência natural de hospedeiros taurinos e zebuínos e a biologia de Boophilus microplus (Canestrini). Ars Veterinaria, 5(1):67-78.

Ojeda-Chi, M. M., Rodríguez-Vivas, R. I., Galindo-Velasco, E., Lezama-Gutiérrez, R. \& Cruz-Vázquez, C. (2011). Control de Rhipicephalus microplus (Acari: Ixodidae) mediante el uso del hongo entomopatógeno Metarhizium anisopliae (Hypocreales: Clavicipitaceae): Revisión. Revista Mexicana de Ciencias Pecuarias, 2(2):177-192.

Paz, G. F., Leite, R. C. \& Oliveira, P. R. (2008). Controle de Rhipicephalus sanguineus (Latreille, 1806)(Acari: Ixodidae) no canil da escola de veterinária da UFMG, Belo Horizonte, Minas Gerais, Brasil. Revista Brasileira de Parasitologia e Veterinária, 1741-44.

Rocha, U. F. (2014). Biologia e controle biológico do carrapato Boophilus microplus (Canestrini). Parasites \& Vectors, 783

Rocha, U. F., Banzatto, D. A., Woelz, C. R., Bechara, G. H., Galluzzi, F. D. \& Garcia, M. C. C. (1984). Ecologia de carrapatos VII-a escassa influência da luz do dia sobre oviposição e embriogênese de Boophilus microplus (Canestrini); equivalência de contagens de larvas e de cascas d'ovos. Semina: Ciências Agrárias, 5(16):5-14.

Rohdich, N., Roepke, R. K. A. \& Zschiesche, E. (2014). Estudo de campo randomizado e cego, controlado e multicêntrico comparando a eficácia e segurança do Bravecto ${ }^{\mathrm{TM}}$ (fluralaner) com o Frontline $^{\mathrm{TM}}$ (fipronil) em cães infestados por pulgas e carrapatos. Parasites \& Vectors, 739.

Santos, A. P. \& Furlong, J. (2002). Competição intraespecífica em Boophilus microplus. Ciência Rural, 32(6): 1033-1038.

Silva, C. R. \& Rocha, E. F. (2004). Estudo sazonal da dinâmica populacional dos estágios parasitários de Boophilus microplus (Canestrini)(Acari: Ixodidae) na pele de hospedeiros da raça Gir. A Hora Veterinária, 24(142):19-22.

Silveira, W. H., Carvalho, G. D. \& Peconick, A. P. (2014). Medidas de controle do carrapato Rhipicephalus microplus: uma breve revisão. PUBVET, 8(Art 1715):1136-1282.

Smith, R. D., Sells, D. M., Stephenson, E. H., Ristic, M. R. \& Huxsoll, D. L. (1976). Development of Ehrlichia canis, causative agent of canine ehrlichiosis, in the tick Rhipicephalus sanguineus and its differentiation from a symbiotic Rickettsia. American Journal of Veterinary Research, 37(2):119126.

Taylor, M. A., Coop, R. L. \& Wall, R. L. (2017). Parasitologia Veterinária. Rio de Janeiro, Brasil: Guanabara Koogan.

Villares, J. B. (1941). Climatologia zootécnica. III. Contribuição ao estudo da resistência e susceptibilidade genética dos bovinos ao Boophilus microplus. Boletim da Indústria Animal, 4(1):6086.

Walker, J. B., Keirans, J. E. \& Horak, I. G. (2005). The genus Rhipicephalus (Acari, Ixodidae): a guide to the brown ticks of the world. London: Cambridge University Press.

Recebido: 10 de maio, 2019.

Aprovado: 3 de junho, 2019.

Publicado: 29 de junho, 2019.

Licenciamento: Este artigo é publicado na modalidade Acesso Aberto sob a licença Creative Commons Atribuição 4.0 (CC-BY 4.0), a qual permite uso irrestrito, distribuição, reprodução em qualquer meio, desde que o autor e a fonte sejam devidamente creditados. 